\title{
Percepção clínica, ética e legal de acadêmicos de Odontologia sobre gerenciamento de resíduos de serviços de saúde
}

\author{
Daylana Pacheco da Silva*, Maysa Luna de Souza**, Graciela Maria Oliveira Sipaúba***, Mariá \\ Soares Leal de Moura****, Valdimar da Silva Valente*****, Carmem Dolores Vilarinho Soares de \\ Moura*****
}

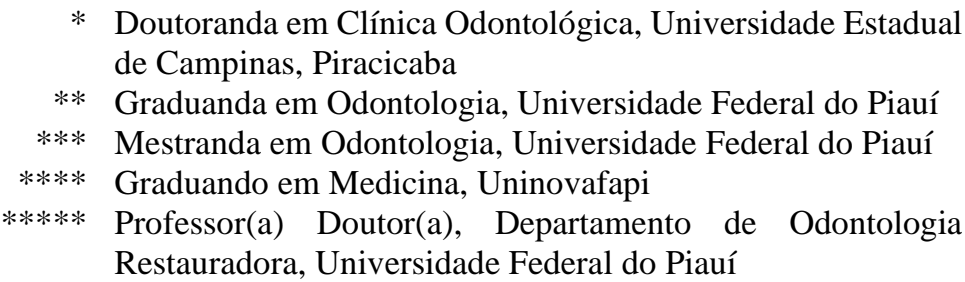

Recebido em 13/11/2018. Aprovado em 24/10/2019.

\begin{abstract}
RESUMO
O objetivo desse estudo foi analisar a percepção clínica, ética e legal de acadêmicos do curso de Odontologia sobre o gerenciamento de Resíduos em Serviços de Saúde (RSS). O estudo foi do tipo observacional transversal, com amostragem censitária de acadêmicos do $8^{\circ}(n=23)$ e $9^{\circ}(n=18)$ períodos. No questionário aplicado abordou-se o processo de produção, manuseio e descarte dos RSS, no que concerne ao gerenciamento, legislação e questões éticas. Os dados foram organizados em planilhas e interpretados por meio de análise descritiva. Sobre as normas vigentes para o gerenciamento de RSS, $82,9 \%$ e $95,1 \%$ dos estudantes afirmaram desconhecer o tratamento/disposição final e regulamento técnico para o gerenciamento de RSS, respectivamente. Em relação ao Plano de Gerenciamento de Resíduos de Serviços de Saúde (PGRSS), 85,4\% não conhecem o Código Penal e 87,8\% não estão familiarizados com o Código Civil. Além de disso, a maioria dos entrevistados desconhecem as Normas da Associação Brasileira de Normas Técnicas $(75,6 \%)$ e as Resoluções do Conselho Nacional do Meio Ambiente $(82,9 \%)$ e da Agência Nacional de Vigilância Sanitária $(95,1 \%)$. Por outro lado, 73,2\% afirmam conhecer os direitos e deveres do cirurgião-dentista, presentes no Código de Ética Odontológica. Quanto à classificação dos resíduos produzidos em ambiente odontológico, 95,1\% responderam corretamente sobre o hipoclorito de sódio e $97,6 \%$ sobre os roletes de algodão e gaze com sangue. Portanto, foi possível concluir que existe conhecimento satisfatório sobre os aspectos clínicos e éticos, entretanto insuficiente quanto às legislações vigentes no Brasil.
\end{abstract}

Descritores: Resíduos de Serviços de Saúde. Estudantes de Odontologia. Minimização de Prejuízos Ambientais. Acondicionamento de Resíduos Sólidos. 


\section{INTRODUÇÃO}

Os desafios que a sociedade moderna enfrenta sobre as dificuldades quanto ao descarte de resíduos compreendem diversos setores, como indústria, trabalho, meio ambiente, saúde $\mathrm{e}$ educação. Portanto, necessitam de um gerenciamento relacionado diretamente com a saúde pública ${ }^{1}$.

Os Resíduos de Serviços de Saúde (RSS) são materiais contaminados por micro-organismos patogênicos, que são produzidos hospitais, clínicas odontológicas, laboratórios, farmácias, clínicas veterinárias e unidades de saúde em geral ${ }^{1}$. Seu descarte deve ser realizado de acordo com a classificação da Agência Nacional de Vigilância Sanitária (ANVISA), considerando a natureza principal do resíduo e o seu potencial de risco, sendo subdivididos em cinco grupos: A biológicos; B - químicos; C - radioativos; D domiciliares; e E - materiais perfurocortantes e escarificantes ${ }^{2}$.

Mesmo que a quantidade do material infectado presente em resíduos odontológicos seja pequena, existe o risco de infecção cruzada e o perigo de contaminação do meio ambiente quando esses resíduos são mal manejados ${ }^{3}$. Assim, o cirurgião-dentista torna-se responsável pela segregação, armazenamento e descarte adequado de todo o resíduo produzido em sem local de trabalho.

Desta forma, o Plano de Gerenciamento de Resíduos de Serviços de Saúde (PGRSS), de acordo com a Resolução do Conselho Nacional do Meio Ambiente (CONAMA) 358/2005, expõe as ações equivalentes ao seu manejo e abrange os aspectos referentes à geração, segregação, acondicionamento, coleta, armazenamento, transporte, tratamento e disposição final, tal como a proteção à saúde pública, assegurando a manutenção da qualidade do meio ambiente ${ }^{4}$.

Dentre as resoluções referentes à saúde e aos resíduos, destacam-se as Normas Brasileiras
Regulamentadoras (NBR) 7500 (símbolos de risco e manuseio para o transporte e armazenamento de materiais), 9191 (sacos plásticos para acondicionamento de lixo), 10004 (riscos à saúde pública e ao meio ambiente sobre os resíduos sólidos) e 12235 (armazenamento de resíduos sólidos perigosos $)^{5-8}$. Além disso, a Agência Nacional de Vigilância Sanitária (ANVISA) estabeleceu, por meio da Resolução da Diretoria Colegiada (RDC) $n^{\circ}$. 33/2003, a regulamentação técnica para o gerenciamento de RSS, determinando também as responsabilidades legais no que se refere ao manuseio, tratamento $\mathrm{e}$ destinação final destes resíduos ${ }^{9}$.

Para a compreensão do gerenciamento de RSS, é necessária uma interferência no processo educacional do curso de Odontologia de forma positiva, a fim de orientar o desenvolvimento do conhecimento teórico-prático durante os estágios clínicos, motivando a capacidade de formação de cirurgiões-dentistas conscientes sobre as responsabilidades ambientais ${ }^{1}$. Portanto, alguns autores investigaram a percepção dos acadêmicos, frente ao descarte de $\mathrm{RSS}^{10-12}$. Diante desta concepção, o presente estudo teve como objetivo analisar a percepção clínica, ética e legal de acadêmicos do Curso de Odontologia da Universidade Federal do Piauí (UFPI) sobre o gerenciamento dos RSS.

\section{METODOLOGIA}

Este estudo foi aprovado pelo Comitê de Ética em Pesquisa da UFPI, com o parecer $n^{\circ}$ 2.602.023. Os participantes foram informados sobre os objetivos da pesquisa e assinaram um termo de consentimento livre e esclarecido (TCLE), obedecendo a Resolução do Conselho Nacional de Saúde CNS no 466/12.

Trata-se de um estudo observacional transversal, conduzido com estudantes do último ano de Odontologia em uma Instituição de Ensino Superior Pública de Teresina/PI. Para coleta de 
dados foi utilizado um questionário desenvolvido e testado por Fernandes ${ }^{13}$, contendo 33 questões abertas e fechadas. Este questionário foi utilizado devido à sua abordagem integral do processo de produção, manuseio e descarte dos resíduos sólidos de saúde, no que concerne ao gerenciamento, legislação e questões éticas. $\mathrm{O}$ questionário foi aplicado por meio de formulário online seguindo rigorosamente a Lei $\mathrm{n}^{\circ} 12.965$, de 23 de abril de 2014, que estabelecem princípios, garantias, direitos e deveres para o uso da internet no Brasil.

A amostra foi censitária e incluiu acadêmicos regularmente matriculados no $8^{\circ}(\mathrm{n}=23)$ e $9^{\circ}(\mathrm{n}=18)$ períodos do Curso de Bacharelado em Odontologia da UFPI. A inclusão dos participantes teve como critério já ter cursado ou estar cursando a disciplina que contempla a temática abordada nesta pesquisa. Os dados foram organizados em planilhas do Programa Microsoft Office Excel 2013 (Microsoft,
Redmond, WA, EUA) e interpretados por meio de análise descritiva.

\section{RESULTADOS}

Dos estudantes que participaram da pesquisa, verificou-se que $70,7 \%(n=29)$ eram do sexo feminino e $90,2 \%$ ( $n=37$ ) encontravam-se na faixa etária entre 20 a 25 anos.

Dentre os entrevistados, todos acreditam que os RSS devam ser coletados por serviço especializado, entretanto apenas quatro estudantes conhecem empresas específicas nesta área. Além disso, 90,2\% acreditam que o adequado tratamento e disposição final dos RSS preservam a saúde pública e a qualidade do meio ambiente. Ainda neste contexto, todos entendem que o correto tratamento e disposição final dos resíduos podem minimizar riscos ocupacionais nos ambientes de trabalho e protegem a saúde do trabalhador (gráfico 1).

Gráfico 1. Conhecimento dos acadêmicos sobre o tratamento e disposição final dos Resíduos de Serviços em Saúde (RSS)

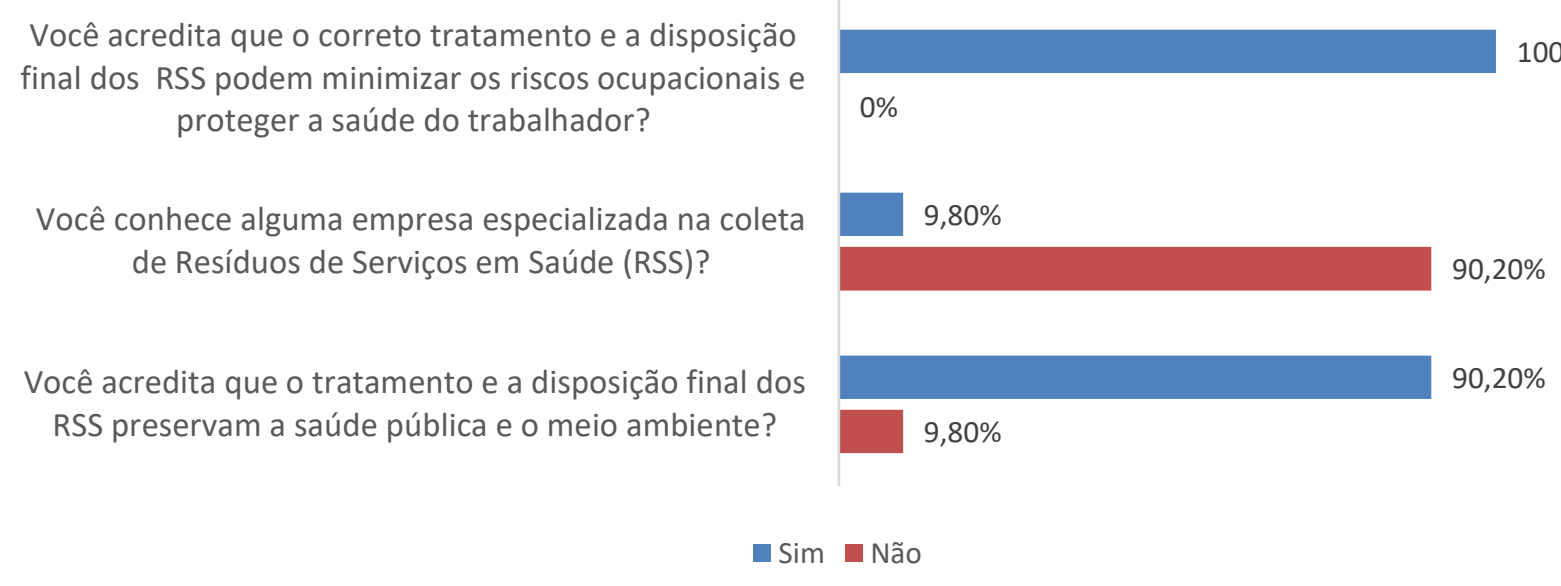

Ao serem questionados sobre os tipos de agentes nocivos à saúde presentes no consultório odontológico, em questão de múltipla escolha, 97,6\% afirmam que sejam agentes biológicos e químicos. Sendo assim, 97,6\% consideram que os RSS devam ser manipulados com Equipamentos de Proteção Individual (EPIs) (tabela 1). 
Tabela 1. Conhecimento sobre Resíduos de Serviços de Saúde

\begin{tabular}{lc}
\hline Variável & $\mathbf{n}(\mathbf{\%})$ \\
\hline Você sabe o que são Resíduos de Serviço de Saúde? & $5(12,2)$ \\
Não & $36(87,8)$ \\
Sim & $40(97,6)$ \\
Qual tipo de agente nocivo à saúde está presente no consultório odontológico?* \\
Biológicos & $40(97,6)$ \\
Químicos & $34(82,9)$ \\
Físicos & $40(97,6)$ \\
Você acredita que os resíduos devam ser manipulados com & EPI? \\
Não & $1(2,4)$ \\
Sim &
\end{tabular}

Sobre o PGRSS, 85,4\% não conhecem o Código Penal em relação às lesões corporais e crimes contra a saúde pública, e consequentemente não estão familiarizados com o Código Civil, no que diz respeito aos atos ilícitos e reparação de danos $(87,8 \%)$. Além de disso, a maioria dos entrevistados desconhecem as Normas da ABNT $(75,6 \%)$ e as Resoluções do CONAMA $(82,9 \%)$ e da ANVISA $(95,1 \%)$. Por outro lado, 73,2\% afirmam conhecer os direitos e deveres do cirurgião-dentista, presentes no Código de Ética Odontológica (tabela 2).

Tabela 2. Conhecimento dos acadêmicos sobre a legislação vigente para o Plano de Gerenciamento de Resíduos de Serviços de Saúde

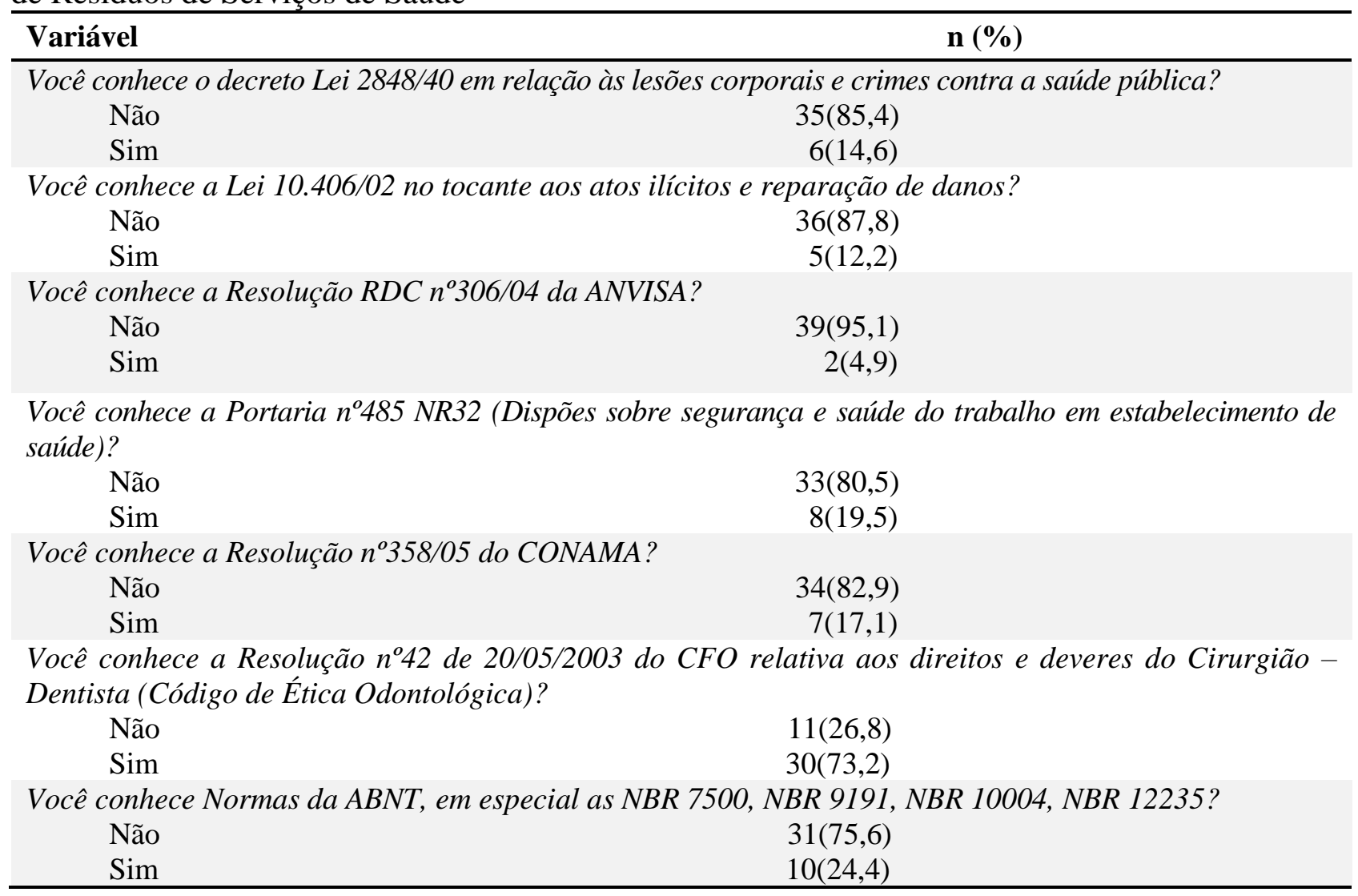


Ao serem questionados sobre a classificação de nove diferentes tipos de resíduos presentes nos consultórios odontológicos, foram obtidos os resultados constantes do gráfico 2.

Em relação à correta classificação do tipo de resíduo, o gesso e invólucros de filmes radiográficos foram as opções que obtiveram maior divergência na categorização. Observou-se que todos os acadêmicos responderam corretamente sobre a forma adequada de descarte de perfurocortantes e líquidos de processamento radiográfico. Sobre o acondicionamento do mercúrio, a maioria dos estudantes (97,6\%) responderam corretamente (tabela 3 ).

Gráfico 2. Distribuição das respostas corretas pertinentes à cada tipo de resíduo gerado pelos consultórios odontológicos

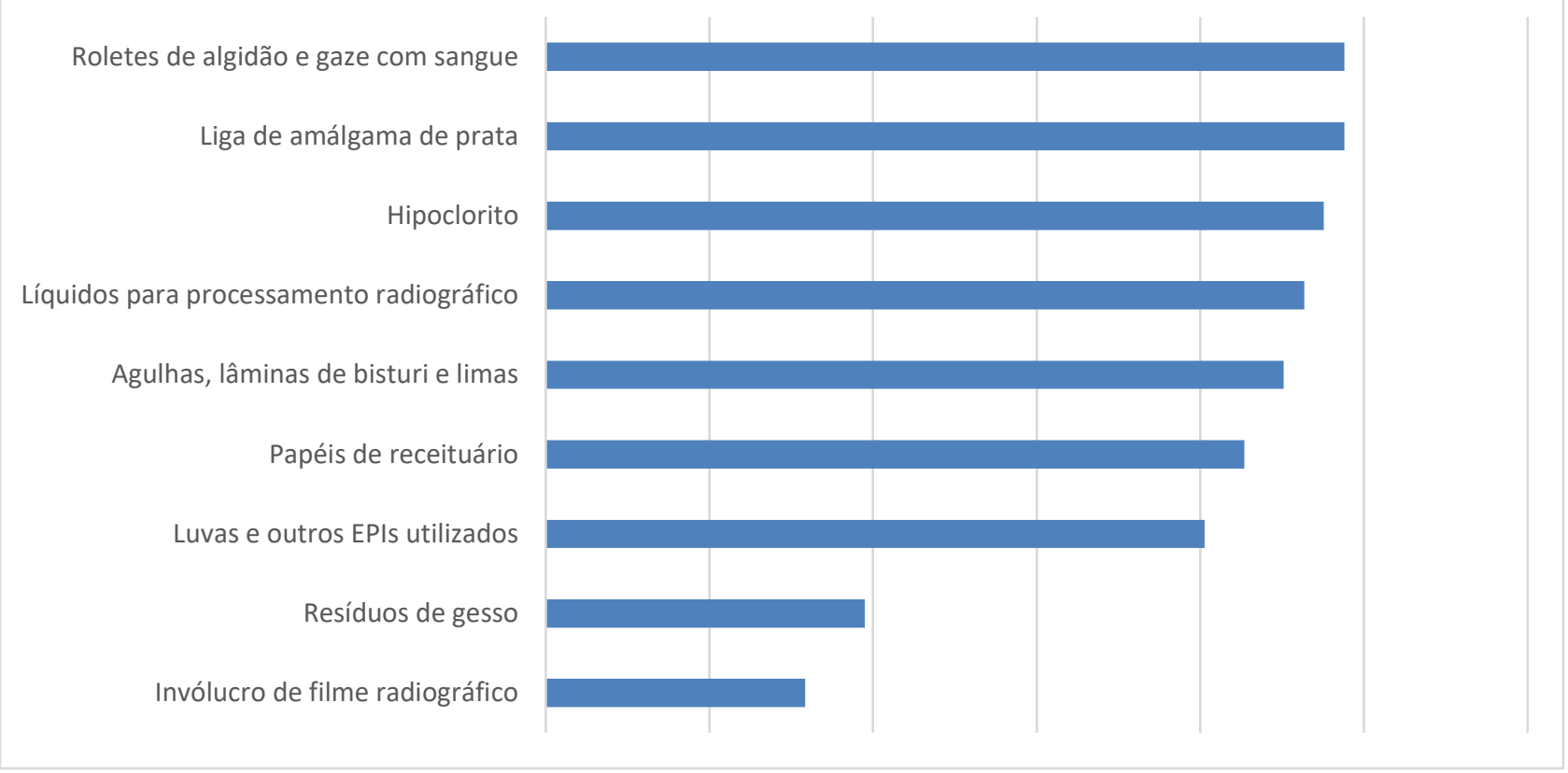

Aos graduandos foi pedido para tecerem comentários sobre a legislação vigente em Odontologia e sobre o conteúdo ministrado durante a graduação, pertinente ao tema do questionário aplicado. Com isso foi obtida uma variedade de respostas, as quais foram classificadas como "positiva", "negativa" ou "não soube opinar". Para os comentários quanto à legislação da profissão considerou-se como positivas respostas como coerente, eficiente e justa; como negativas burocrática, necessita de melhorias e desatualizada. Quanto ao conteúdo curricular considerou-se como positivo discorrer sobre um dos temas abordados e negativo quando continham os termos superficial, não foi ministrado e pouco abordado (tabela 4). 
Tabela 3. Conhecimento dos estudantes sobre o Plano de gerenciamento de Resíduos de Serviços de Saúde

\begin{tabular}{lc}
\hline Variável & $\mathbf{n}(\%)$ \\
\hline $\begin{array}{l}\text { Sobre o acondicionamento de mercúrio residual, você acredita que deva ser em recipiente inquebrável com } \\
\text { tampa, contendo água no seu interior? }\end{array}$ & $40(97,6)$ \\
Sim & $1(2,4)$ \\
Não & $41(100)$ \\
A forma correta de descarte dos líquidos revelador e fixador de radiografias é acondicioná-los em frascos \\
resistentes com tampa e posteriormente encaminhá-los a para coleta especial? \\
Sim \\
Não & - \\
Em relação ao recipiente para acondicionamento do material perfurocortante desprezado (agulha, lâminas \\
de bisturi etc.), você acredita que ele deva ser colocado em recipientes com paredes rígidas, impermeável, \\
resistente a ruptura e rotulado? \\
Sim \\
Não \\
\hline
\end{tabular}

Tabela 4. Distribuição dos casos de acordo com as respostas abertas

\begin{tabular}{lc}
\hline Variável & $\mathbf{n}(\%)$ \\
\hline Percepção sobre a legislação vigente em Odontologia & $17(41,5)$ \\
Positiva & $14(34,1)$ \\
Negativa & $10(12,4)$ \\
Não soube opinar & $13(31,7)$ \\
Percepção sobre o conteúdo ministrado durante a graduação pertinente ao tema do questionário aplicado \\
Positiva & $22(53,7)$ \\
Negativa & $6(14,6)$ \\
Não soube opinar &
\end{tabular}

\section{DISCUSSÃO}

A falta de padronização no gerenciamento dos RSS pode causar comprometimentos à saúde humana e ao meio ambiente ${ }^{14}$. Sendo assim, todos os estabelecimentos que oferecem serviços em saúde devem elaborar seu próprio plano de gerenciamento de descarte, com o objetivo de minimizar a produção de resíduos e proporcionar uma condução segura e eficiente deste processo $^{15}$. Assim, os resultados deste estudo mostram que apesar dos acadêmicos acreditarem que o tratamento dos resíduos seja capaz de preservar a saúde pública e a qualidade do meio ambiente, apenas $9,8 \%$ conhece uma empresa de coleta especializada na área. Este fato evidencia que o conhecimento teórico dos estudantes não é vivenciado na prática.

No Código de Ética Odontológica ${ }^{16}$, a Resolução $n^{\circ}$ 071/2006 expressa o dever dos CD de se recusar a exercer a profissão em âmbito público ou privado quando as condições de trabalho não sejam dignas, seguras e salubres, bem como zelar pela saúde e dignidade dos pacientes. Como observado, a maioria dos discentes afirmam conhecer seus direitos e deveres, mostrando a capacidade de interferir no ambiente de trabalho, como também de identificar condições impróprias para o gerenciamento de RSS.

A legislação atribui ao estabelecimento de serviço de saúde a responsabilidade pelo descarte apropriado do RSS, e aos profissionais o seu 
gerenciamento. Nos estudos de Borges et al. $(2016)^{17}$ e Alves et al. $(2016)^{18}$ foram observadas falhas em várias fases da gestão de resíduos, não atendendo aos princípios preconizados pelas legislações vigentes. Além disso, Alves et al. $(2016)^{18}$ mostram o desconhecimento dos profissionais sobre as resoluções que regem o gerenciamento de RSS. Este fato influencia diretamente as gestões futuras na formação de profissionais conscientes dos seus deveres.

A quantidade de resíduos potencialmente infectantes gerados nos consultórios odontológicos é expressivamente menor se comparada aos hospitais, no entanto são gerados resíduos químicos de mercúrio, glutaraldeído, hipoclorito de sódio, revelador e fixador de radiografias ${ }^{19}$. Assim, a maioria dos entrevistados considera que os principais RSS sejam químicos e físicos, devendo ser manipulados com EPIs.

Em relação à correta classificação dos tipos de resíduos, constatou-se que os resíduos de gesso e os invólucros de filme radiográfico foram os itens que tiveram maior porcentagem de erro de categorização. Os resíduos de gesso não apresentam risco biológico ou químico à saúde ou ao meio ambiente, podendo ser equiparados aos resíduos domiciliares. Já o invólucro de filmes radiográficos é considerado um resíduo químico, pois a lâminas de chumbo, ao ser descartado de forma incorreta, pode resultar em contaminação do solo e de águas subterrâneas ${ }^{20}$. Desta forma, as lâminas de chumbo devem ser acondicionadas separadamente e identificadas com o símbolo de risco associado, de acordo com a NBR 7500, e posteriormente devem ser encaminhadas ao Aterro Sanitário Industrial para Resíduos Perigosos ${ }^{21}$.

Em relação ao correto armazenamento dos resíduos perfurocortantes e mercúrio residual, verificou-se assentimento diante do risco à saúde proveniente destes resíduos, constatando-se um alto percentual de acertos, assim como nos estudos de Pereira et al. $(2015)^{10}$ e Melo et al. (2008) ${ }^{22}$. Este fato pode ser atribuído à maior ênfase dada pelos professores durante a graduação sobre estes materiais, provavelmente devido ao alto índice de acidentes perfurocortantes relatados na literatura ${ }^{23}$. Já o mercúrio é uma substância reconhecidamente tóxica, tanto que foi a primeira substância química a ser submetida a uma legislação para controle da exposição ${ }^{24}$. Assim, de acordo com RDC no 173/17, é proibido em todo o território nacional a fabricação, importação e comercialização do mercúrio e do pó para liga de amálgama não encapsulado, utilizados para fins odontológicos ${ }^{25}$.

Sobre o descarte dos líquidos revelador e fixador radiográficos, Pereira et al. $(2015)^{10}$ e Fernandes (2009) ${ }^{13}$ obtiveram resultados contrários aos do presente estudo. Portanto, destaca-se um ponto positivo para a sociedade, já que os problemas ambientais causados por esses contaminantes vão desde a contaminação das águas até a sua deposição em áreas nativas ${ }^{26}$.

Sobre a percepção dos estudantes com relação à legislação vigente em Odontologia e conteúdos ministrados durante a graduação, um número expressivo deles mostrou-se insatisfeito, sugerindo reformulação e atualização do conteúdo, dando ênfase aos direitos e deveres do cirurgião-dentista no âmbito do gerenciamento dos RSS. Já em relação aos conteúdos ministrados, os acadêmicos afirmaram que muitos não foram abordados durante o curso, ou foram apresentados com deficiência. Neste aspecto, o fato evidencia a necessidade de se ampliar debates no ambiente acadêmico com o intuito de elevar o conhecimento dos estudantes sobre o tema. Diante disto, o colegiado de curso foi alertado para a importância de revisão no conteúdo programático das disciplinas que abordam questões legais e civis.

Essa deficiência no conhecimento em âmbito acadêmico se espelha na atuação profissional. Como observado por diversos autores ${ }^{20,26,27,29-31}$, os responsáveis pelo gerenciamento necessitam de maior treinamento referente ao tema, já que este 
processo envolve inúmeras falhas.

Diante destes resultados, sugerimos que pesquisas relacionadas ao gerenciamento de RSS, aspectos legais e civis, sejam realizadas em outros cursos de Odontologia e Unidades Básicas de Saúde, para o conhecimento da realidade de outras regiões. Além disso, este tema deve ser abordado de forma mais objetiva e eficiente na graduação, para que os futuros profissionais participem ativamente do processo de gestão do tratamento dos resíduos.

\section{CONCLUSÃO}

O conhecimento dos estudantes da presente amostra sobre o gerenciamento de Resíduos em Serviços de Saúde é satisfatório quanto aos aspectos clínicos e éticos, entretanto insuficiente quanto aos aspectos legais.

\section{ABSTRACT \\ Dental Students' Clinical, Ethical, and Legal Perception on Healthcare Waste Management}

The aim of this study was to analyze the dental students' clinical, ethical and legal perception on the management of health services waste (HSW). This was a cross-sectional observational study, with census sampling of students from the 8th $(n=23)$ and 9th $(n=18)$ terms. The applied questionnaire addressed the process of production, handling and disposal of HSW, regarding management, legislation and ethical issues. Data were organized in spreadsheets and interpreted by descriptive analysis. Regarding the current rules for the management of WHS, $82.9 \%$ and $95.1 \%$ of the students stated that they did not know the final treatment/disposition and technical regulation for HSW management, respectively. Regarding the Health Services Waste Management Plan (HSWMP), 85.4\% did not know the Criminal Code and $87.8 \%$ were not familiar with the Civil Code. In addition, most respondents were unaware of the principles of the Brazilian Association of Technical Standards (75.6\%) and the Resolutions of the National Environmental Council (82.9\%) and the National Health Surveillance Agency (95.1\%). On the other hand, $73.2 \%$ claimed to know the rights and duties of the dental surgeon, present in the Code of Dental Ethics. Regarding the classification of residues produced in a dental environment, $95.1 \%$ answered correctly about sodium hypochlorite and $97.6 \%$ about cotton and blood gauze rollers. Therefore, it was possible to conclude that there is satisfactory knowledge about the clinical and ethical aspects, however insufficient regarding the current legislation in Brazil.

Descriptors: Health Services Waste. Dental Students. Minimization of Environmental Damage. Solid Waste Packaging.

\section{REFERÊNCIAS}

1. Leal CAG. Biossegurança e gerenciamento de resíduos de serviços de saúde: a importância na formação do profissional da Odontologia na perspectiva da saúde humana e ambiental. Rev ABENO. 2015; 15(2):82-94.

2. Ministério da Saúde. Agência Nacional de Vigilância Sanitária - ANVISA. Resolução da Diretoria Colegiada no 306 de 07 de dezembro de 2004. Brasília (DF); 2004.

3. Hidalgo LRC, Garbin AJI, Rovida TAS, Garbin CAS. Gerenciamento de resíduos odontológicos no serviço público. Rev Odontol UNESP. 2013; 42(4):243-50.

4. Ferreira ID. Gerenciamento de resíduos de serviços de saúde: Orientações para os serviços em Odontologia. [Trabalho de conclusão de curso]. Juiz de Fora: Universidade Federal de Juiz de Fora; 2014.

5. Associação Brasileira de Normas Técnicas (ABNT). NBR 7500. Símbolos de risco e manuseio para transporte e armazenamento de material. Rio de Janeiro (RJ); 2000.

6. Associação Brasileira de Normas Técnicas (ABNT). NBR 9191. Sacos plásticos para acondicionamento de lixo - Requisitos e métodos de ensaio. Rio de Janeiro (RJ); 
2000.

7. Associação Brasileira de Normas Técnicas (ABNT). NBR 10.004. Resíduos sólidos classificação. Rio de Janeiro (RJ); 2004.

8. Associação Brasileira de Normas Técnicas (ABNT). NBR 12235. Armazenamento de resíduos sólidos perigosos. Rio de Janeiro (RJ); 1992.

9. Ministério da Saúde. Agência Nacional de Vigilância Sanitária - ANVISA. Manual de gerenciamento de resíduos de serviços de saúde. Brasília (DF); 2006.

10. Pereira KCR, Locks KW, Squizatto LM, Silva Junior MF, Miclos PV. Resíduos dos serviços de saúde: Conhecimento sobre a geração e responsabilidade dos formandos em Odontologia das faculdades de Santa Catarina. Arq Odontol. 2015; 51(2): 88-95.

11. Faye D, Mbacke LCM, Kanouté A. Waste management from dental care in the health districts of Dakar, Senegal. Med Sante Trop. 2014; 24: 403-8.

12. Gomes AMP, Garbin AJI, Arcieri RM, Rovida TAS, Garbin CAS. Sustentabilidade ambiental: gerenciamento de resíduos odontológicos no Serviço Público. Rev Cuba Estomatol. 2017;54(2).

13. Fernandes MM. Conhecimento dos formandos em odontologia sobre o plano de gerenciamento de resíduos de serviços de saúde - aspectos éticos e legais. [Trabalho de conclusão de curso]. Piracicaba: Universidade Estadual de Campinas; 2009.

14. Krishnappa K, Sreekantaiah P, Hiremath SS, Thapsey H, Shivraj NS, Murthy NS. Quantification of dental health care waste generated among private dental practices in Bengaluru City. J Int Oral Health. 2015; 7(6):84-87.

15. Maders GR, Cunha HFA. Análise da gestão e gerenciamento dos resíduos de serviços de saúde (RSS) do Hospital de Emergência de
Macapá, Amapá, Brasil. Eng Sanit Ambient. 2015; 20(3):379-388.

16. Conselho Federal de Odontologia. Resolução $\mathrm{n}^{0} 71$, de 06 de junho de 2006. Rio de Janeiro (RJ); 2003.

17. Borges AMM, Timóteo JEN, Brasil MA, Brito AB, Pinto AGA, Fonseca FLA. Gerenciamento de resíduos em serviços de saúde do Sertão Nordestino. SMA. 2016;5(2):93-105.

18. Alves SC, Gonçalves MF, Monterosso EP, Godecke MV. Gerenciamento de Resíduos de Saúde: estudo de caso em estabelecimentos públicos municipais de Pelotas, RS. REGET. 2016; 20(1):105-14.

19. Danaei M, Karimzadeh P, Momeni M, Palenik CJ, Nayebi M, Keshavarzi V, Askarian M. The management of dental waste in dental offices and clinics in Shiraz, Southern Iran. Int J Occup Environ Med. 2014;5(1):18-23.

20. Singh H, Bhaskar DJ, Dalai DR, Rehman R, Khan M. Dental Biomedical Waste Management. Int J Sci Stud. 2014;2(4):66-8.

21. Ministério do Meio Ambiente. Conselho Nacional do Meio Ambiente. Resolução CONAMA n ${ }^{\circ} .358$ de 29 de abril de 2005. Brasília (DF); 2005.

22. Melo LSV, Radicchi, R, Carvalho CM, Rodrigues V. Aspectos odontolegais da insalubridade em odontologia. RGO. 2008; 56(2):143-149.

23. Nascimento LS, Assunção LRS, Silva Júnior NG, Pedreira EN, Silva RLC. Acidentes com pérfuro-cortantes na Faculdade de Odontologia da UFPA: Visualização de um Cenário. Rev Odontol Bras Central. 2012;21(56).

24. Jesus LF, Marinha MS, Moreira FR. Amálgama dentário: fonte de contaminação por mercúrio para a Odontologia e para o meio ambiente. Cad. Saúde Colet. 
2010;18(4): 509-15.

25. Ministério da Saúde. Agência Nacional de Vigilância Sanitária - ANVISA. Resolução ANVISA n ${ }^{\circ} 173$ de 15 de setembro de 2017. Brasília (DF); 2017.

26. Santos JMR, Gomes AT. Gerenciamento de efluentes de serviço de radiologia: inquérito realizado em três centros de saúde da região dos lagos estado do Rio de Janeiro. A Biomed Brasiliensia. 2017;8(1).

27. Momeni H, Fard SFT, Arefinejad A, Afzali A, Tabeli F, Salmani ER. Composition, production rate and management of dental solid waste in 2017 in Birjand, Iran. Iran. Int J Occup Environ Med. 2018;9:52-60.

28. Kontogianni S, Xirogiannopoulou A, Karagiannidis A. Investigating solid waste production and associated management practices in private dental units. Waste Management. 2008;28:1441-8.
29. Daoua MH, Karamb R, Khalil S, Mawla D. Current status of dental waste management in Lebanon. Environ. Nanotec Monit.2015;4:1-5.

30. Lopes DAC, Calabrese L. Gerenciamento de resíduos de serviço de saúde. Rev Saúde. 2016;(10):1-2.

31. Rosa FR, Machado EL, Moraes JAR, Kist LT. Gerenciamento e quantificação dos resíduos dos serviços de saúde: estudo de caso. Tecno-Lógica. 2016; 20(2):111-7.

\section{Correspondência para:}

Daylana Pacheco da Silva

e-mail: daylanapachecos@gmail.com

Universidade Federal do Piauí

Rua Miosótis, 1135 - Jóquei

64048-908 Teresina/PI 